Dominador B. Toral, MD

Chris Robinson D. Laganao, MD

Department of Ear Nose Throat - Head and Neck Surgery Southern Philippines Medical Center
Correspondence: Dr. Chris Robinson D. Laganao Department of Ear, Nose, Throat - Head and Neck Surgery Southern Philippines Medical Center

Bajada, Davao City 8000

Philippines

Phone: +639165395589 / +639238353675

Email:dmc_ent@yahoo.com

The authors declared that this presents original material that is not being considered for publication or has not been published or accepted for publication elsewhere or in part, in print or electronic media; that the requirements for authorship have been met by all the authors, and the author believes that the manuscript represents honest work.

Disclosures: The authors signed a disclosure that there are no financial or other (including personal) relationship, intellectual passion, political or religious beliefs, and institutional affiliations that might led to conflict of interest.

Presented at the Philippine Society of Otolaryngology Head and Neck Surgery Descriptive Research Contest 2020 (2nd Place), 21 October 2020

\section{Classification and Stages of Middle Ear Cholesteatoma at the Southern Philippines Medical Center Using the European Academy of Otology and Neurotology / Japan Otological Society (EAONO / JOS) System}

Results: Of the 42 cases evaluated, congenital cholesteatoma was seen in 4 while acquired cholesteatoma was noted in 38 , (further subdivided into 34 retraction pocket cholesteatoma and 4 non-retraction pocket/traumatic cholesteatoma). A majority (57\%) had Stage II cholesteatoma (mass occupying at least two sub-sites in the middle ear) at the time of surgery. Eight (19\%) had stage I cholesteatoma (confined to one sub-site), five (12\%) had stage III cholesteatoma evidenced by extracranial complications such as subperiosteal abscess and erosion of the semicircular canals. Stage IV cholesteatoma was seen in $5(12 \%)$ presenting with intracranial abscess. Canal wall down mastoidectomy was the most common surgical approach performed. The sinus tympani $\left(S_{2}\right)$ was the most commonly involved difficult to access site across all classifications of middle ear cholesteatoma (60\%).

Conclusion: Our study provided an initial profile of the stages and severity of middle ear cholesteatoma in our institution based on actual surgical approaches. Such a profile can be the nidus for a database that can help us to understand disease prevalence and compare local surgical practices with those in the international community.

Keywords: cholesteatoma, aural; cholesteatoma, middle ear; cholesteatoma, middle ear*/ classification; cholesteatoma, middle ear*/diagnosis; cholesteatoma, middle ear*/surgery 
Cholesteatoma is benign growth of keratin tissue in the middle ear cavity. Typically, patients present with chronic ear discharge and sometimes, hearing loss, and imbalance, depending on the anatomical extent of bony erosion by the cholesteatoma. 'The global estimate of the incidence of middle ear cholesteatoma reaches up to approximately 9 to 12.6 cases per 100,000 adults and from 3 to 15 cases per 100,000 children. ${ }^{2}$ In the Philippines, the disease prevalence is estimated at $4.2 \%$ but is feared to be much higher though underreported due to health economic reasons. ${ }^{3}$

Attempts have been made in the past to classify or categorize cholesteatoma and propose a clinically acceptable and practical staging system. ${ }^{4}$ In 2017, the European Academy of Otology and Neurotology and the Japan Otological Society published the EAONO/JOS Joint Consensus Statements on the Definitions, Classification and Staging of Middle Ear Cholesteatoma in an attempt to finally create a uniform system of staging that would be recognized globally. ${ }^{1}$ However, to the best of our knowledge, based on a search of HERDIN Plus, the ASEAN Citation Index, and the Global Index Medicus - Western Pacific Region Index Medicus, the EANO/JOS definitions, classifications, and staging have not been applied locally in the Philippines.

This study used the EANO/JOS classification system to determine the stage of middle ear cholesteatoma of patients who underwent middle ear surgery at the Southern Philippines Medical Center from January to December 2019 in order to provide a profile of the severity of cases and the surgical approaches used to address the extent of the disease in our institution. It is hoped that with the uniformity of the classification and staging system, surgical approaches and results can be compared as the disease process is understood even further.

\section{METHODS}

With Department of Health XI Cluster Ethics Review Committee (DOH XI CERC) approval (P20021701), this case series searched the Southern Philippines Medical Center hospital information database from January 2019 to December 2019 for cases of cholesteatoma and the respective patient charts were retrieved from the medical records section. Records of all patients who underwent middle ear surgery for cholesteatoma during the study period were considered for inclusion. Charts of patients who were admitted for reasons other than surgery of the middle ear, or who were re-operated on the ipsilateral side were excluded.

Completeness of each chart was assessed in terms of history information sheet, diagnostic examinations, surgical memorandum, and intraoperative findings. Results of ancillary procedures and diagnostic examinations not present in the chart (such as audiology records) were retrieved from the appropriate department archive.

The demographic profile (age, gender, otologic history including the chief complaint and onset of symptoms), relevant diagnostic

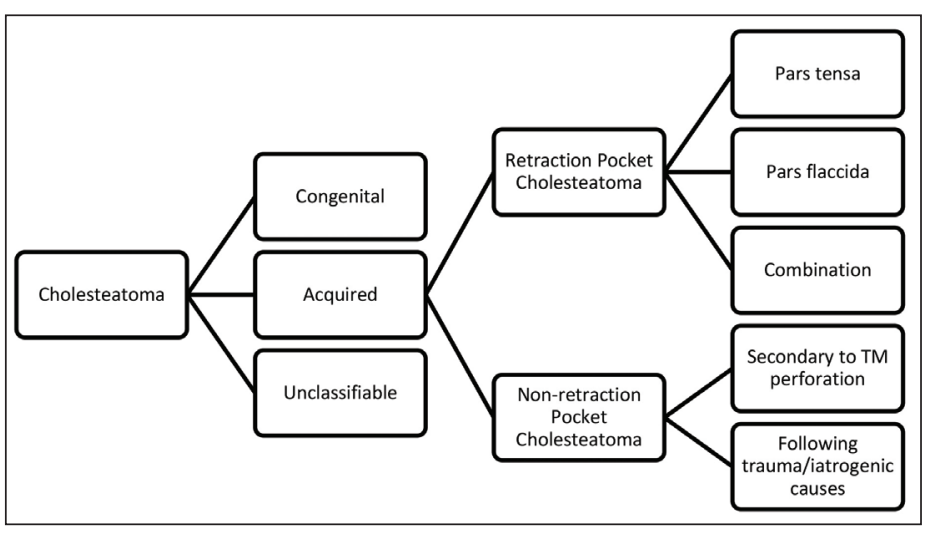

Figure 1. Schematic drawing of the clinical classification of middle ear cholesteatoma used in the EAONO/JOS consensus statement (adapted from reference 1).

procedures, surgical memorandum and technique were extracted from each chart and filled into a data gathering tool specifically constructed for this study.

The stage of middle ear cholesteatoma was determined on each chart using the EAONO/JOS system. Based on this system, middle ear cholesteatoma was staged I when only one primary site was affected. For pars flaccida cholesteatoma, stage I meant that the cholesteatoma formation was limited only to the attic. For congenital cholesteatoma, the mass should be seen behind an intact tympanic membrane. If cholesteatoma formation was due to pars tensa perforation, stage I denoted presence of cholesteatoma only in the tympanic cavity. Stage II cholesteatoma meant more than 1 primary site was affected. For pars flaccida cholesteatoma, stage II meant that either the tympanic cavity or mastoid antrum was also filled with keratin. For pars tensa cholesteatoma, stage II meant involvement of the attic and difficult access sites such as the supratubal recess and sinus tympani. Stage III cholesteatoma meant that extracranial extension was evident intraoperatively, and manifestations of extracranial extension may have been present preoperatively (e.g. ipsilateral facial nerve palsy, dizziness and balance disorders) as well as abscess formation in the parotid, posterior auricular area, and sternocleidomastoid muscle. Stage IV cholesteatoma implied associated intracranial complications such as abscess and purulent meningitis, otitic hydrocephalus and lateral sinus thrombosis. (Figure 1)

The surgical memoranda and operative techniques recorded the extent of cholesteatoma and evaluated important anatomic structures in reference to the EAONO/JOS classification system which includes the difficult site (S), tympanic cavity $(\mathrm{T})$, attic $(\mathrm{A})$, and mastoid $(\mathrm{M})$. The difficult access sites are further subdivided into $\mathrm{S} 1$, supra tubal recess and S2, sinus tympani. (Figure 2) These findings were collated in our data gathering tool.

Data was tabulated and means and frequencies were computed using Microsoft Excel ${ }^{\circ}$ for Mac Version 16.16.27 (Microsoft Corp. Redmond, WA, USA). 


\section{ORIGINAL ARTICLES}

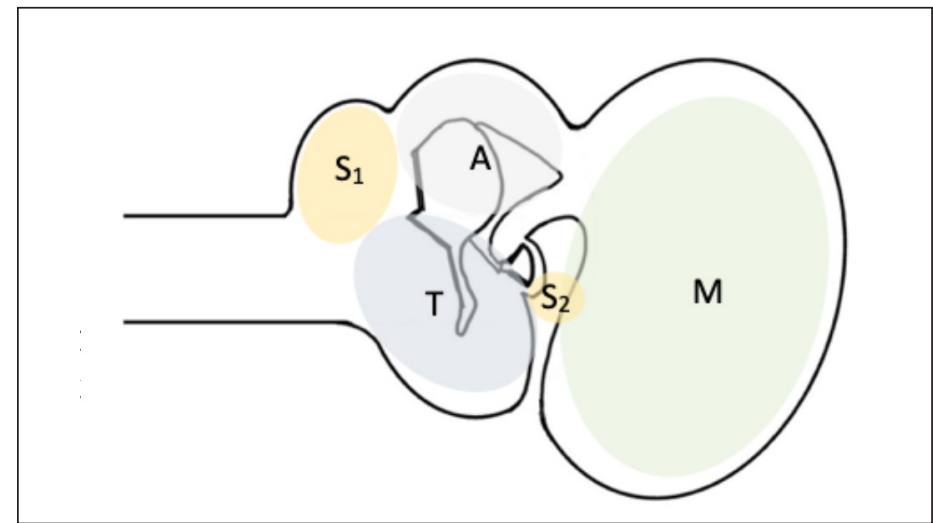

Figure 2. Schematic drawing of the clinical classification of middle ear cholesteatoma used in the EAONO/JOS consensus statement (adapted from reference 1).

\section{RESULTS}

Our initial hospital database search revealed 96 records of patients with cholesteatoma admitted during the study period. Fifty (50) records of those admitted due to a medical reason other than surgery of the middle ear were excluded. Of the 46 remaining records of mastoidectomy done in 2019, four more were excluded due to reoperation on the ipsilateral ear. The 42 remaining charts were checked for completeness including the surgical memorandum and intraoperative findings. Ancillary and diagnostic procedures not present in the charts were retrieved from the audiology records kept in the department. All 42 records qualified and were included in this study.

Twenty-nine (69\%) were in the 18 to 40 -year-old age bracket. There were eight (19\%) minors ( $<18$ years old) and five $(12 \%)$ older than 40. There were 20 males (48\%) and 22 females (52\%) females. Slightly over half were single $(22 ; 52 \%)$.

The most common chief complaint was ear discharge $(20 ; 48 \%)$ followed by hearing problems $(18 ; 43 \%)$ and ear pain $(4 ; 10 \%)$. Twenty five $(60 \%)$ reported symptoms for more than 5 years, $14(33 \%)$ had symptoms for more than one year, and three (7\%) had symptoms for less than a year.

None of the patients had normal hearing thresholds. Most (15; $36 \%)$ had moderate conductive hearing loss, nine (21\%) had severe conductive hearing loss, and three (7\%) had mild conductive hearing loss. Twelve (29\%) had profound sensorineural hearing loss, with no cases of mild, moderate or severe sensorineural hearing loss. There were two (5\%) cases of moderate mixed hearing loss and one (2\%) case of severe mixed hearing loss. Thirty-eight (90\%) had flat (Type B) tympanometry results, three (7\%) had "Type A" tympanograms, and one (2\%) had a "Type As" tympanogram.

There were four (9\%) congenital cholesteatomas (based on preoperative description of intact tympanic membrane with no history of trauma or ear discharge) and $38(90 \%)$ acquired cholesteatomas. Of these, retraction pocket cholesteatoma was seen in $34(81 \%)$, while non-retraction pocket cholesteatoma was noted in the remaining four (9\%).

In terms of the type and approach of middle ear surgery, two out of four cases (50\%) of congenital cholesteatoma were managed thru canal wall down mastoidectomy, while two other cases were through transcanal approach. All four cases of congenital cholesteatoma were stage III, of which three (75\%) presented with post auricular subperiosteal abscess formation.

Among the 34 cases of retraction pocket cholesteatoma, canal wall down mastoidectomy was the most common surgical approach (22 out of 34 cases). Canal wall up mastoidectomy was performed in 5 cases of Stage I and in 4 cases of Stage II retraction pocket cholesteatoma. Transcanal approach was also performed in 2 cases of Stage I and 1 case of Stage II retraction pocket cholesteatoma.

For the four non-retraction pocket cholesteatomas, a trans-canal approach was performed for one Stage I and one Stage II case each, a canal wall up procedure for the other stage II case, and a canal wall down approach for the single Stage IV case.

The sinus tympani $\left(S_{2}\right)$ was the most commonly involved difficult to access site across all the classifications of middle ear cholesteatoma. It was noted to be involved in 25 cases (60\%) overall. The supra-tubal recess $\left(\mathrm{S}_{1}\right)$ was involved in 14 cases $(33 \%)$.

Overall, 24 cases (57\%) were Stage II (cholesteatoma occupying at least two subsites in the middle ear cleft). Stage I cholesteatoma (confined to one subsite only) was seen in 8 patients (19\%), and Stage III (extracranial complications) and IV (intracranial complications) cholesteatomas were seen in 5 patients (12\%) each.

\section{DISCUSSION}

A cholesteatoma is a benign mass in the middle ear cavity composed of keratin debris capable of erosion of adjacent bony structures. The extent of cholesteatoma dictates the morbidity it can cause and may range from hearing loss, ear discharge, and ear pain to intracranial extension. In 2017, the European Academy of Otology and Neurotology (EAONO) and the Committee on Nomenclature of the Japan Otological Society (JOS) agreed on a practical system of classification generally accepted by otogists/neurotologists internationally. Even so, the application of such a classification is still in its early stages.

The majority of patients (69\%) in this present study were in the 18 to 40-year-old range. This finding parallels the finding that most patients (60\%) had long-standing ear complaints. Their age on admission may be indicative of the period when they finally gave significance to their ear complaints or achieved sufficient economic means to seek medical consult. ${ }^{2,5}$

The nature of cholesteatoma is often insidious. In most cases, the ear complaints start as a minimal ear discomfort associated with upper respiratory tract infections that often resolve spontaneously. Over 
succeeding episodes of recurrent nasal congestion, infection may set in and discharge from the ear can be a harbinger of a more chronic otologic pathology. Repetitive infection can cause mucosal inflammation in the middle ear mucosa that favors the formation of cholesteatoma. ${ }^{6,7}$ Ear discharge $(48 \%)$ followed by hearing problems $(43 \%)$ were common chief complaints of patients coming to the hospital. Although ear pain may comprise only $10 \%$ as chief complaint overall, the presence of ear pain was more often associated with more advanced staged middle ear cholesteatoma. Patients with extracranial and intracranial complications of cholesteatoma present symptoms such as posterior auricular abscess (Stage III), or neck pain from meningitis (Stage IV).

Hearing difficulty on the background of cholesteatoma is an important predictor of the prognosis of the patient. When cholesteatoma has eroded the middle ear ossicles, mechanical conduction of sound would be affected. This can be seen as a conductive hearing loss on the Pure Tone Audiogram (PTA) and varies depending on the severity i.e. mild, moderate, severe. When the cholesteatoma is large enough to erode even the bony cochlea, the PTA would reflect a profound hearing loss.? In this study, most patients (36\%) had a moderate conductive hearing loss, while profound hearing loss was found in $29 \%$. Theoretically, profound hearing loss can be difficult to reverse and the indication of surgery is geared towards disease elimination and not necessarily hearing conservation. Meanwhile, mixed hearing loss is a combination of both conductive and sensorineural causes. Mixed hearing loss was documented in 3 patients in this study, 2 (5\%) moderate and $1(2 \%)$ severe. An organic sensorineural hearing loss seen especially among individuals above 40 years old coupled with the formation of cholesteatoma produces a mixed-type hearing loss on PTA. 6,7

Furthermore, tympanometry studies showed fairly predictable results. Tympanometry is a test that determines the mobility of the tympanic membrane in response to pressure changes. It is designated as " $A$ " when the tympanic membrane is mobile, "As" when it is stiff, "Ad" when it is lax, " $B$ " when it is flat/fixed, and " $C$ " when it is retracted? A type $B$ tympanogram is more consistent with cholesteatoma because it implies that the tympanic membrane does not move because of the space-occupying lesion behind it. In this study, an overwhelming $90 \%$ of the ears had type B tympanograms. Although not found in our sample, a type $C$ tympanogram suggests Eustachian tube dysfunction, an important early pathophysiologic culprit of cholesteatoma formation. ${ }^{6,7}$ The participants in our study had more advanced disease than the earlier-stage eustachian tube dysfunction that would have been reflected by a type $C$ tympanogram.

Findings of this study support our experience that patients with cholesteatoma seek consult only when symptoms become troublesome, which coincides clinically with Stage II disease. Symptom tolerance is a significant factor that may explain why early stage disease is uncommonly seen in our department. Indeed, Stage III and Stage IV diseases often present with sequela of long standing ear disease such as meningitis or abscesses in the posterior auricular area and neck.

Our results may vary from those of other institutions should they replicate the methods used in our series. Factors such as the general economic status of their sample population may alter the frequency distribution of cholesteatoma stages. When ear complaints are not given priority until symptoms of intracranial symptoms are evident, then Stage I and II diseases may not even be documented.

Nevertheless, this study needs to be continued so that future cases of cholesteatoma are appropriately documented. A more appropriate study design would be a prospective multicenter study, using a uniform comprehensive case report form. Even if an international database is not yet available, we highly recommend that the EANO/JOS definition, classification, and staging system be used in all otorhinolaryngology residency training institutions in the country to provide comparative data on disease stages, surgical approaches and outcomes.

In conclusion, our study provided an initial general profile of the stages and severity of cholesteatoma in our institution based on actual surgical approaches. Such a classification and staging of middle ear cholesteatoma can serve as the nidus for a database of cholesteatoma classification and staging in our institution that can help us to understand the disease prevalence and compare local surgical practices with those in the international community.

\section{REFERENCES}

1. Yung $M$, Tono $T$, Olszewska $E$, Yamamoto $Y$, Sudhoff $H$, Sakagami $M$, et al. EAONO/JOS Joint Consensus Statements on the Definitions, Classification and Staging of Middle Ear Cholesteatoma. J Int Adv Otol. 2017 Apr;13(1):1-8. DOI: 10.5152/iao.2017.3363 PubMed PMID: 28059056.

2. Kuo C-L, Shiao A-S, Yung M, Sakagami M, Sudhoff H, Wang C-H, et al. Updates and knowledge gaps in cholesteatoma research. BioMed Res Int. 2015;2015:854024. DOI: 10.1155/2015/854024 PubMed PMID: 25866816 PubMed Central PMCID: PMC4381684.

3. Chan KH, Dreith S, Uhler KM, Tallo V, Lucero M, De Jesus J, Simões E. Large-scale otoscopic and audiometric population assessment: A pilot study. Int J Pediatr Otorhinolaryngol. 2019 Feb;117:148-152. DOI: 10.1016/j.ijporl.2018.11.033 PubMed PMID: 30579070 PubMed Central PMCID: PMC6338678.

4. Linder TE, Shah S, Martha AS, Röösli C, Emmett SD. Introducing the "ChOLE" classification and its comparison to the EAONO/JOS consensus classification for cholesteatoma staging. Otol Neurotol. 2019 Jan; 40(1):63-72. DOI: 10.1097/MAO.00000000000002039 PubMed PMID: 30339650

5. Tono T, Sakagami M, Kojima H, Yamamoto $Y$, Matsuda K, Komori $M$, et al. Staging and classification criteria for middle ear cholesteatoma proposed by the Japan Otological Society. Auris Nasus Larynx. 2016;44(135-140). DOI: 10.1016/j.anl.2016.06.012 PubMed PMID: 27616746.

6. Chole R. Chronic otitis media, mastoiditis, and petrositis. In: Flint PW, Haughey B, Lund V, Niparko K, Lesperance M, Thomas Robbins K, Regan Thomas J, editors. Cummings OtolaryngologyHead and Neck Surgery. 6th ed. Philadelphia: Elsevier Inc; 2015.p.2139-2155.

7. Lee KJ. Audiology. In: Lee KJ, Chan Y, Das S, editors. Essential Otolaryngology Head and Neck Surgery. 10th ed. New York: McGraw-Hill; 2012. p.24-67. 